\title{
Pase de guardia: información relevante y toma de decisiones en clínica médica. Estudio prospectivo
}

\author{
Andrea Vázquez, Graciela de Carlo, Pablo Marchetti, Diego Monopoli, Beatriz Montico, Néstor Morgulis, \\ Pedro del Río
}

Introducción. El pase de guardia es una actividad médica en la que se transfiere información y responsabilidad entre profesionales en situaciones de discontinuidad o transiciones en el cuidado de los pacientes. Los pases de guardia son fuente de errores médicos, a pesar de lo cual la programación formal en la competencia específica está ausente en los currículos de las residencias médicas. En este sentido, implementamos el proyecto educativo 'Pase de guardia oral y escrito en la residencia de clínica médica'.

Materiales y métodos. Definimos el constructo 'información relevante' a partir de cinco ítems, uno sistémico y cuatro cognitivos. Se analizó la prevalencia de los déficits de información relevante y su repercusión sobre la práctica clínica.

Resultados. En 230 protocolos de guardia, la prevalencia de déficits de información relevante fue del 31,3\% ( $n=72)$ y afectó tanto al ítem sistémico (11\%) como a los ítems con contenidos sustantivos (20\%). Con información relevante, las conductas activas fueron del 34,6\%, y las pasivas, del 65,4\%; con déficits de información relevante, las activas fueron del $13,9 \%$, y las pasivas, del 86,1\%. Estas diferencias fueron significativas $(p<0,001)$.

Conclusiones. Los déficits de información relevante tienen alta prevalencia en los pases de guardia y favorecen los errores por omisión. La mayor parte de los errores médicos reconocen fallos en habilidades cognitivas propias del razonamiento clínico de médicos en formación (errores cognitivos), por lo que se hace necesario incorporar el pase de guardia oral y supervisado al currículo de la residencia de medicina interna.

Servicio de Clínica Médica. Departamento de Medicina. Sanatorio Guemes. Hospital Universitario. Universidad de Buenos Aires. Buenos Aires, Argentina.

Correspondencia:

Dr. Pedro del Río. P .Goyena, 564, 4. - A. C1424 BSS. Buenos Aires, Argentina.

E-mail:

podelrio@gmail.com

Agradecimientos:

A la Dra. M. Khoury, por su lectura crítica y sugerencias, y a la licenciada S. de Gutsztat, por su asesoría pedagógica

C 2011 Educación Médica

Palabras clave. Competencia comunicativa. Educación en el internado y la residencia. Educación médica. Error médico.

\section{Handoffs: relevant information and decision making in internal medicine. A prospective study}

Introduction. Handoffs are medical activity which transfers information and responsibility among professionals in situations of discontinuity or transitions in patient care. Handoffs are source of medical errors and adverse events, which despite the formal programming of specific competencies are absent in the curricula of medical residencies. In this sense, we implemented the educational project 'Oral and written handoffs in internal medicine residency program'.

Materials and methods. We defined the parameter relevant information with a systemic item and four other cognitive items; we assess the prevalence of relevant information deficits and the effects on the clinical practice in a prospective study.

Results. In 230 protocols the prevalence of relevant information deficits was 31.3\% ( $n=72$ ) and affected both, systemic item (11\%) as the cognitive items (20\%). With relevant information, active behaviors were $34.6 \%$ and passive $65.4 \%$; with relevant information deficits, the active behaviors were $13.9 \%$ and $86.1 \%$ passive respectively, this difference was significant $(p<0.001)$.

Conclusions. We conclude that relevant information deficit is highly prevalent in the handoffs and results in errors of omission. The majority of medical errors recognize flaws in their own cognitive skills (cognitive errors) of clinical reasoning of physicians in training, so it is necessary to incorporate the oral and supervised handoffs at the residency curriculum.

Key words. Communication skills. Internship and residency education. Medical education. Medical errors.

\section{Introducción}

El pase de guardia (PG) constituye una actividad clínica compleja en la que se transfiere informa- ción y responsabilidad médica entre profesionales. Ambas actividades resultan vulnerables al error médico, por lo que el desarrollo de la competencia comunicativa y el profesionalismo médico son re- 
Tabla I. Ítems de información relevante.

\begin{tabular}{ll}
\hline Indicador & Descriptor \\
\hline $\begin{array}{l}\text { 1. Datos de filiación y de ingreso } \\
\text { 2. Contexto clínico en el ingreso actual }\end{array}$ & Definir el contexto clínico del ingreso actual \\
\hline $\begin{array}{l}\text { 3. Problemas activos (del día) } \\
\text { Controles vitales actualizados }\end{array}$ & $\begin{array}{c}\text { Identificar problemas del día } \\
\text { Actualizar controles vitales o emplear escalas pertinentes } \\
\text { (p. ej., Glasgow en pacientes neurológicos) }\end{array}$ \\
\hline $\begin{array}{l}\text { 4. Evolución clínica previsible } \\
\text { Anticipar eventos contingentes y conductas a adoptar } \\
\text { (si... entonces...) }\end{array}$ \\
$\begin{array}{l}\text { De diagnóstico } \\
\text { De tratamiento } \\
\text { De control clínico }\end{array}$ \\
$\begin{array}{l}\text { Ejecutar maniobras, interpretar resultados, } \\
\text { realizar interconsultas }\end{array}$ \\
$\begin{array}{l}\text { Activa } \\
\text { Pasiva }\end{array}$
\end{tabular}

quisitos clave para brindar una asistencia médica segura [1].

Los PG se realizan toda vez que se producen discontinuidades en la atención médica: cambios de guardia, rotaciones médicas, derivaciones entre servicios o instituciones [2]... Estas transiciones son propicias para las distorsiones $\mathrm{u}$ omisiones en la información o en la transferencia de responsabilidades; los errores pueden reconocer componentes médicos propiamente dichos [3] o componentes sistémicos de la organización o institución [4] que exigen acciones médicas de último momento para evitar incidentes adversos. Esta situación se torna más crítica cuando se trata de pacientes complejos [5], si las discontinuidades son múltiples [6] o si intervienen médicos en formación [7].

A pesar de su importancia, el PG como competencia específica permanece ignorado en el diseño de los currículos académicos. En Estados Unidos, el $60 \%$ de los programas de las residencias acreditadas en medicina interna no incluyen entre sus objetivos curriculares el PG como competencia específica, y en el $27 \%$ de las residencias, los residentes no recibieron entrenamiento ni supervisión apropiada relacionada con el PG [8]. En Argentina, las nociones del PG se adquieren empíricamente como 'practicantes de guardia. En los currículos de los postulantes a la residencia de clínica médica, el 53\% reconocen haber participado en guardias extracurriculares en calidad de 'practicante de guardia'; la experiencia se autoeva- luó como positiva, aunque todos los entrevistados admitieron no haber tenido instrucción ni entrenamiento específicos relacionados con el PG.

La residencia médica parece ser la oportunidad impostergable para afrontar el tema de los PG ya que los residentes asumen por primera vez la atención supervisada de pacientes. Para caracterizar los contenidos del PG desarrollamos el concepto de información relevante (IR). El objetivo principal de nuestro estudio fue, por una parte, caracterizar la competencia comunicativa de los médicos residentes en el PG, y por otra, relacionar el factor de riesgo déficit de información relevante (DIR) con las conductas adoptadas durante la guardia.

\section{Información relevante}

Aunque no se han establecido estándares de calidad respecto al contenido conceptual de IR, dos características esenciales serían: que sea pertinente respecto a los hechos (relevancia referencial) o respecto a posibles conductas (relevancia pragmática). Ambos aspectos deberían ser evaluables en la práctica clínica; para ello apelamos a la construcción de un indicador operacional de IR que incorpore criterios adoptados en la práctica clínica propia o de otros autores. Cada ítem consta de un indicador que es evaluado según el criterio de relevancia estandarizado en el descriptor (Tabla I).

\section{Descripción de los ítems}

- Item 1. Identificación personal: tiene como objetivo la detección de errores sistémicos [4] vinculados con la identificación del paciente.

- Ítem 2. Definir el contexto clínico: evalúa el proceso cognitivo que implica seleccionar datos relevantes, estructurar y relacionar conceptos.

- Ítem 3. Identificar el problema activo $[9,10]$ : evalúa la capacidad cognitiva para identificar el problema activo, esto es, actualizar el contexto clínico y establecer prioridades respecto a las comorbilidades.

- Ítem 4. Evolución clínica anticipada [10]: evalúa la capacidad de prever o sugerir conductas diagnósticas y terapéuticas según la evolución anticipada del paciente.

- Ítem 5. Tareas pendientes [7]: evalúa la habilidad para ejecutar o interpretar actividades pendientes, como recepción de estudios de laboratorio en general (bioquímicos, radiológicos, ecográficos), interconsultas, maniobras instrumentales, etc.

- Ítem 6. Toma de decisiones [7]: evalúa el desempeño acorde con la información disponible. Conduc- 
ta activa significa modificar o implementar nuevas conductas: conducta pasiva significa no innovar.

\section{Materiales y métodos}

\section{Diseño del estudio}

En una cohorte de residentes del Servicio de Clínica Médica se estudió de forma observacional y prospectiva la competencia comunicacional en el PG mediante la utilización del constructo IR.

El Servicio de Clínica Médica tiene una capacidad instalada de 264 camas y el ingreso promedio de pacientes es de 1.200 al mes. El plantel médico estable está integrado por 16 médicos exresidentes, y la residencia, por 32 médicos. Todos los residentes de primer a cuarto año cumplen ocho guardias activas al mes; no realizan guardias pasivas y se respetan los intervalos de 48 horas libres de guardia. Además, 1014 médicos externos (residentes o exresidentes) concurren a realizar guardias nocturnas o los fines de semana. Los médicos externos cubren aproximadamente el $25 \%$ del tiempo total de guardia en planta.

Todos los residentes de clínica médica presentes durante el período de estudio participaron en el proyecto educativo denominado 'Pase de guardia seguro', por tratarse de una exigencia curricular. El método de estudio y sus objetivos se informaron con antelación. No fue necesario cumplir con otros requerimientos éticos.

\section{Implementación del PG oral y escrito}

Los pacientes incluidos en el PG fueron seleccionados con la supervisión del médico de planta a cargo y se emplearon criterios clínicos de gravedad o inestabilidad clínica aceptados por la práctica clínica.

Se realizan tres sesiones de PG al día. En el PG vespertino, con la asistencia de residentes, jefe de Residentes y miembros del equipo, se discute con criterios docentes/asistenciales cada protocolo del PG y se los distribuye entre los médicos de guardia. En el PG nocturno, algunos protocolos se transfieren a médicos externos. En el PG matutino participan los médicos de guardia, los médicos del equipo y residentes. El PG escrito consiste en un formulario con pautas orientadoras del contenido y se adoptó como estrategia de enseñanza con un doble objetivo: sistematizar los contenidos del PG y controlar la gestión. Los ítems o atributos a completar fueron:

- Item 1: datos de filiación, ubicación del paciente, sello y rúbrica del residente que intervino.

- Item 2: contexto clínico.
- Item 3: problema activo y actualización de parámetros.

- Ítem 4: evolución anticipada previsible.

- Item 5: ejecución de tareas pendientes.

- Ítem 6: conductas activas o pasivas.

La selección de pacientes (casos) para su inclusión en el PG fue realizada por cada residente con supervisión del médico de planta; los ítems 1, 2, 3 y 4 fueron completados por el residente a cargo del paciente, y los ítems 5 y 6 , por el médico de guardia. Las actualizaciones en el protocolo de PG no reemplazaron a las de la historia clínica. Al finalizar su guardia, cada residente entregaba los protocolos utilizados. Cada protocolo de guardia correspondía a un paciente al día.

\section{Instrumento de evaluación}

Cada protocolo fue evaluado de forma independiente por dos de los autores (B.M., P.D.R.). Se utilizó la tabla I como lista de cotejo para evaluar los contenidos del PG escrito, a la que se le adjuntó una escala sumativa de Likert. Los atributos de cada ítem se adjudicaron de forma independiente y en caso de discrepancia la puntuación se acordó por consenso. Cada uno de los tres meses se evaluó por separado para analizar la estabilidad de las puntuaciones obtenidas.

\section{Escala de medición}

La escala de Likert consta de categorías ordinales y se adoptaron los cinco atributos siguientes: muy incompleto, 1 punto; incompleto, 2 puntos; suficiente, 3 puntos; completo, 4 puntos, y muy completo, 5 puntos. En el análisis posterior, la escala ordinal se transformó en nominal al considerar los ítems con puntuación 3 , 4 y 5 como IR, y los ítems con puntuación 1 y 2, como DIR.

\section{Análisis estadístico}

Tanto la IR como el DIR se expresaron en porcentaje. Se estimaron los intervalos de confianza para proporciones. La comparación entre grupos con variables categóricas se analizó con la prueba de chi al cuadrado. Los valores de $p<0,05$ se consideraron significativos.

\section{Resultados}

El PG seguro se implementó entre septiembre del 2007 y marzo del 2008. De forma aleatoria se selec- 
Figura 1. Frecuencia relativa de los déficits con información relevante (DIR) discriminados por ítem (1, 2, $3,4,5,6)$ y global $(7)$.

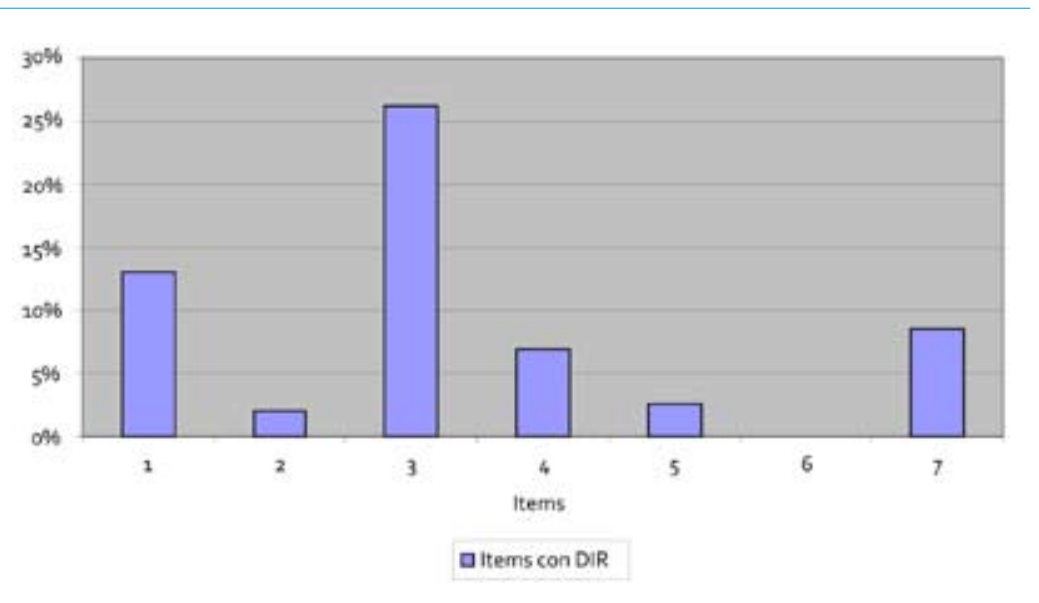

Tabla II. Información relevante (IR) y déficit de IR (DIR) global y por ítem ( $n=230)$.

\begin{tabular}{lcccc}
\hline & DIR & IR & DIR (\%) & DIR (IC 95\%) \\
\hline Ítem 1 & 30 & 200 & 13,0 & $12,9-13,0$ \\
\hline Ítem 2 & 5 & 225 & 2,1 & $2,0-2,1$ \\
\hline Ítem 3 & 42 & 188 & 26,2 & $20,0-31,0$ \\
\hline Ítem 4 & 16 & 214 & 6,9 & $6,8-6,9$ \\
\hline Ítem 5 & 6 & 224 & 2,6 & $2,6-2,6$ \\
\hline Global & 99 & 1.051 & 8,6 &
\end{tabular}

IC 95\%: intervalo de confianza del 95\%.

cionaron para su análisis tres de los siete meses. Se utilizó la tabla de números aleatorios para seleccionar 100 de 149, 80 de 153 y 50 de 109 protocolos, respectivamente.

\section{Control de gestión}

En 219 protocolos de guardia se registraron 632 actualizaciones, esto es, cada protocolo de guardia se actualizó en promedio tres veces. No se completaron 11 protocolos $(4,7 \%)$.

\section{Protocolos con DIR}

La prevalencia de protocolos con DIR fue del 31,3\% (72/230). En estos 72 protocolos se detectaron 99 ítems con DIR, y el 8,6\% de estos protocolos tuvieron más de un ítem con DIR.

\section{Ítems}

En la tabla II y la figura 1 se muestra la prevalencia de DIR global y discriminada por ítem. El ítem con mayor prevalencia de DIR fue el 3, seguido del 1 y el 4. El rango del intervalo de confianza refleja la significación estadística de todos los DIR detectados.

\section{Relación entre DIR y toma de decisiones durante la guardia}

Los datos de la tabla de contingencia $2 \times 2$ muestran la relación existente entre fallos en la comunicación y las conductas adoptadas (Tabla III). En este análisis quedaron excluidos los ítems 1 . Se excluyeron los 11 protocolos que estaban incompletos.

En los 219 protocolos restantes se observó que con IR las conductas fueron activas en el 34,6\% (61/176) de las ocasiones, mientras que con DIR las conductas activas fueron del 13,9\% (6/43). Esta diferencia fue estadísticamente significativa $(p<0,001)$ (Fig. 2).

También surge de la misma tabla que hubo un $19,6 \%$ (43/219) de conductas adoptadas carente de IR: en 6 de 67 conductas activas y en 37 de 152 conductas pasivas.

\section{Discusión}

Los tres principales hallazgos de este estudio muestran que:

- Los DIR son frecuentes en los PG (31\% de los protocolos); afectan tanto a los ítems relacionados con la gestión (11\%) como a los ítems relacionados con contenidos sustantivos (20\%).

- Los DIR desalentaron las conductas activas, favoreciendo los errores médicos o eventos adversos por omisión.

- Los DIR (resultados) están condicionados por errores cognitivos (procesos) propios de médicos en formación.

La prevalencia de protocolos con DIR en nuestra serie fue del $31,3 \%$, que coincide con otro estudio de diseño similar. Borowitz et al [11] analizaron en un estudio de diseño similar la efectividad del PG en una guardia de pediatría; evaluado por los propios residentes, reconocieron que el 31\% de los PG omitieron información necesaria para tomar decisiones durante la guardia. Los déficits prevalentes correspondieron a nuestros ítems 2,3 y 4 , y los autores explican el fallo por la falta de programas formales de entrenamiento. Analizar los DIR en cada uno de los 
ítems puede contribuir a diseñar mejor los contenidos de los programas de formación específica.

\section{Ítem 1. Identificación personal}

Este tipo de errores médicos dan lugar a incidentes adversos tales como la administración de medicamentos, transfusiones o procedimientos invasivos, en el paciente equivocado [12]. Se desconoce en nuestro medio la prevalencia de incidentes adversos relacionados con errores en la identificación de pacientes; posiblemente pueda atribuirse a dos razones: carencia de cultura institucional en la denuncia voluntaria u obligatoria de incidentes adversos y riesgo de judicialización de la práctica. Aunque no todos los errores médicos de este tipo se transforman en incidentes adversos debido a la acción médica de último momento, en nuestra experiencia detectamos un 13\% (30/230) de los protocolos con errores en la identificación. Una táctica destinada a prevenirlos o detectarlos es la implementación de estructuras organizativas más seguras [6]; la protocolización del PG en formato electrónico con campos específicos obligatorios parece ser una iniciativa apropiada [13-15].

Otra iniciativa dirigida a fortalecer la habilidad comunicativa sería el desarrollo de la cultura del trabajo en equipo. En un análisis retrospectivo de 240 demandas de mala praxis en las que estuvieron involucrados médicos en formación, los errores médicos relacionados con el trabajo en equipo estuvieron presentes en el $70 \%$ de los casos, siendo la falta de supervisión y la carencia de PG los predominantes [7]. Otras deficiencias grupales fueron líneas de responsabilidad confusas y conflictos personales. La expresión 'quinta disciplina' se refiere a la cultura organizacional del trabajo en equipo [16]. El trabajo en equipo constituye una habilidad social cuyo aprendizaje es cooperativo y se ejerce en un contexto clínico. El aprendizaje grupal es interactivo y el factor aglutinador es la comunicación (diálogo, discusión). Este objetivo curricular exige cambios en la actitud personal; la demanda verdadera es una transformación, en el ethos de la residencia, del rendimiento individual clásico al desempeño en equipo [17].

\section{Ítem 2. Definir el contexto clínico}

Se trata de evaluar la capacidad de plantear problemas siguiendo el aforismo 'problema bien planteado, problema medio resuelto'. En nuestra serie es baja la prevalencia de DIR relacionados con 'plantear el problema clínico'. Este resultado puede atribuirse en gran parte a la tutoría ejercida por los
Figura 2. Conductas médicas activas y pasivas según el tipo de información disponible: información relevante frente a déficits de información relevante.

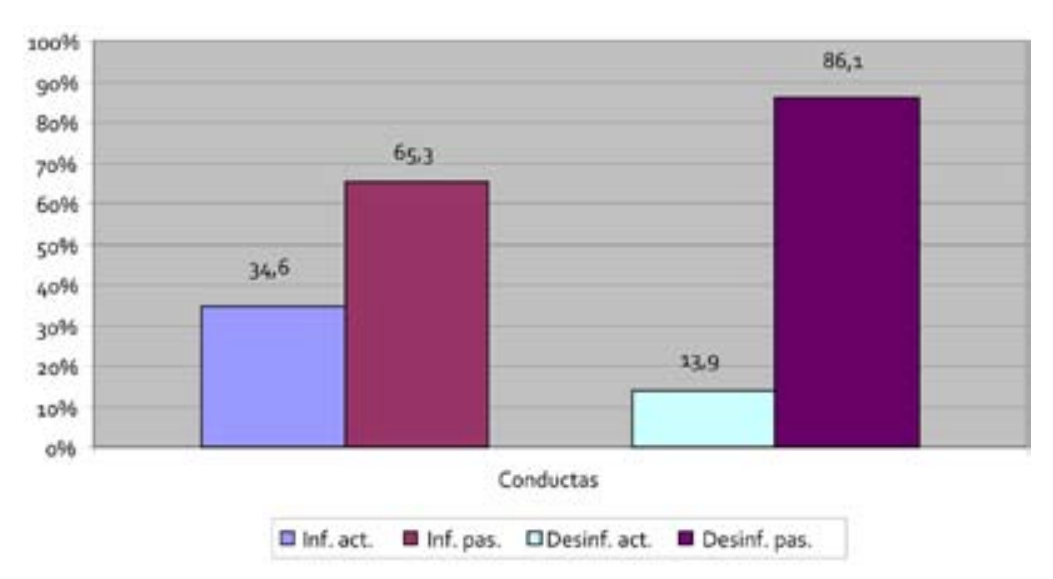

Tabla III. Toma de decisiones con (IR) o sin información relevante (DIR).

\begin{tabular}{lcccc}
\hline & $\begin{array}{c}\text { Conductas } \\
\text { activas }\end{array}$ & $\begin{array}{c}\text { Conductas } \\
\text { pasivas }\end{array}$ & $\begin{array}{c}\text { Total de } \\
\text { protocolos }\end{array}$ & $\begin{array}{c}\text { Conductas } \\
\text { activas (\%) }\end{array}$ \\
\hline IR (Likert 3, 4, 5) & 61 & 115 & 176 & 34,6 \\
\hline DIR (Likert 1, 2) & 6 & 37 & 43 & 13,9 \\
\hline $\begin{array}{l}\text { Posibles errores } \\
\text { adjudicables a DIR (\%) }\end{array}$ & $8,9(6 / 67)$ & $24,3(37 / 152)$ & $19,6(43 / 219)$ & \\
\hline
\end{tabular}

médicos de planta en la selección y discusión de los criterios de inclusión de pacientes en el PG. Definir el contexto clínico es una habilidad cognitiva que supone representación conceptual y razonamiento clínico, que se adquiere gradualmente tras atravesar etapas de empirismo, memorización y generalización inductiva [18]. Por ejemplo, ante un paciente que cursa el primer día de un postoperatorio con hiperkalemia, pH ácido, afectación de la funcionalidad renal, ecocardiograma anormal y antecedentes de diabetes surgen, en primera instancia, términos descriptivos u observacionales como hiperkalemia y pH ácido, a partir de los cuales se representa conceptualmente como 'acidosis metabólica' y se establecen relaciones con la funcionalidad renal o los niveles de glucemia o el daño tisular. Aprender a seleccionar los datos, especificar categorías semánticas [19], como acidosis metabólica, y establecer relaciones entre conceptos (filtrado glomerular, cetoacidosis) es la puesta en práctica de razonamiento clínico más avanzado $[18,20]$. 
En definitiva, la baja incidencia de errores en este ítem nos confirma la importancia del PG oral o 'frente a frente' tutorizado como el principal instrumento de comunicación del PG, y debe considerarse el patrón de referencia respecto a las demás tecnologías [21]. La estrategia pedagógica durante el PG consistiría en 'solicitar aclaraciones': precisar, sugerir, formular, reformular criterios diagnósticos o de tratamiento...

Al mismo tiempo, se debe estar alerta sobre los sesgos cognitivos que pueden afectar la práctica clínica [22]. El sesgo de 'encuadre' o 'contextual' (framing effect) se produce cada vez que el receptor del PG jerarquiza los datos recibidos en función del contexto planteado por el emisor, es decir, el receptor jerarquiza lo que fue jerarquizado por el emisor. En el ejemplo anterior, aceptar el contexto 'hiperkalemia postoperatoria' sin otras consideraciones diagnósticas alternativas lo hace solidario tanto en los aciertos como en los errores, lo que no es recomendable en un PG que tiene objetivos asistenciales y educacionales.

\section{Ítem 3. Identificar el problema activo}

Este ítem es uno de los más afectados por los DIR en la experiencia propia y de otros $[9,10]$. En nuestra serie afectó al 20-31\% de los protocolos de guardia.

Son varios los errores cognitivos que contribuyen a este DIR. En primer lugar, es muy frecuente la descripción anacrónica del contexto clínico (día de ingreso), sin tener en cuenta los acontecimientos sucedidos en el curso del ingreso, sesgo reconocido como 'anclaje heurístico'. El contexto clínico actualizado requiere incorporar nuevas categorías semánticas (cambio, comparación) y las relaciones de aumento, disminución, estático, dinámico, estabilidad, inestabilidad... El segundo error consiste en no diferenciar comorbilidades de problemas activos, y el tercero, en omitir datos empíricos que serán de imprescindible referencia durante la guardia, como solicitar 'control neurológico' en un paciente con traumatismo craneal sin explicitar la escala de Glasgow inicial. En este sentido, es recomendable promover la incorporación de escalas con validez externa comprobada (Glasgow, APACHE II, TISS).

Desde el punto de vista pedagógico, también este ítem requiere el planteamiento de objetivos cognitivos, tanto conceptuales como reflexivos [15]. La estrategia cognitiva ha consistido en incorporar al PG nuevas categorías semánticas: lista de problemas, jerarquización de problemas, causa, efecto, comorbilidades y problemas activos.

\section{Ítem 4. Evolución clínica anticipada}

Cerca del 7\% de los protocolos tienen DIR en este ítem. El error típico puede ejemplificarse como sigue: se solicita 'controlar la fiebre' en un paciente neutropénico; esto puede significar tratamiento sintomático, retirar un catéter, iniciar tratamiento empírico, etc. La conducta adecuada puede ser prevista o adelantada según criterio del médico de planta o residente que tiene una comprensión situada del paciente, con lo que se evitan decisiones erróneas por médicos no familiarizados con el problema del paciente [2], pero además se evita la pérdida de tiempo que supone leer una historia clínica completa para tener una comprensión cabal de la situación.

Desde el punto de vista cognitivo, este tipo de pensamiento de ir más allá de la información o de los hechos es una habilidad generativa que permite comprender situaciones-problema y, a partir de ellas, inferir y predecir otras. En parte se adquiere con la experiencia asistencial, ya que se trata de una forma de generalización inductiva $[9,10,19]$.

\section{Ítem 5. Tareas pendientes}

Este ítem estuvo afectado del DIR en el 2,6\% de los protocolos. El error o limitación cognitiva más frecuente fue 'transcribir datos' sin comprender o interpretar su relevancia clínica. En otros casos, el error consistió en elegir un contexto clínico anacrónico (anclaje heurístico); por ejemplo, adjudicar el incremento de la glucemia en un diabético como propia de la diabetes, sin evaluar la aparición reciente de signos de sepsis, como fiebre e hipotensión arterial. La instrucción debería promocionar 'pensar sobre los datos', apelando a la selección, organización y recontextualización clínica de éstos.

\section{Ítem 6. Déficit de información}

relevante y toma de decisiones

Nuestros resultados muestran que los DIR condicionan la conducta de los residentes. El decremento del 34,6\% al 13,9\% de las conductas activas se puede interpretar como potenciales errores médicos por omisión; se trata principalmente de diferir u omitir indicaciones o procedimientos [19]. Respecto a la fuente potencial de eventos adversos secundarios a DIR, se puede reconocer que un $19,6 \%$ de las decisiones médicas activas o pasivas durante la guardia se tomaron sin información suficiente (Tabla III).

Por otra parte, es interesante señalar que la sola práctica del PG escrito/oral no atenuó de forma automática la incidencia de DIR durante el seguimien- 
to longitudinal, por lo que se hace necesario planificar intervenciones pedagógicas específicas dirigidas a mejorar tanto la gestión como los contenidos del PG. Respeto a la gestión, se ha sugerido la incorporación de tecnologías informáticas para mejorar la eficacia y calidad del PG al centralizar y organizar la información, pero no se deberían alentar falsas expectativas respecto a sus efectos sobre los procesos cognitivos implicados en la toma de decisiones. Puesto en perspectiva, por razones educativas y asistenciales, el PG oral debe incorporarse al currículo de la residencia médica [23]. Las exigencias mínimas comprenden: práctica sistemática del PG en un espacio físico adecuado, con privacidad; comunicación interactiva con jerarquización de funciones y trabajo en equipo, y promover la denuncia de no conformidades o incidentes adversos. Respecto a los contenidos o IR se debería alentar la utilización de estrategias cognitivas supervisadas que promuevan habilidades de pensamiento generales y específicas.

\section{Bibliografía}

1. Arora VM, Johnson JK, Meltzer DO, Humphrey HJ. A theoretical framework and competency-based approach to improving handoff. Qual Saf Health Care 2008; 17: 11-4.

2. Petersen, LA, Brennan TA, O'Neil AC, Cook EF, Lee TH. Does housestaff discontinuity of care increase the risk for preventable adverse events? Ann Intern Med 1994; 121: 866-72.

3. Thomas EJ, Petersen L. Measuring errors and adverse events in health care. J Gen Intern Med 2003; 18: 61-7.

4. Cook RI, Render M, Woods DD. Gaps in the continuity of care and progress on patient safety. BMJ 2000; 320: 791-4.

5. Berkenstadt H, Haviv Y, Tuval A, Shemesh Y, Megrill A, Perry A, et al. Improving handoff communication in critical care. Chest 2008; 134: 158-62.

6. Nolan TW. System changes to improve patient safety. BMJ 2000; 320: 771-3.

7. Singh H, Thomas EJ, Petersen L, Studdert DM. Medical errors involving trainees. A study of closed malpractice claims from five insurers. Arch Intern Med 2007; 167: 2030-6.

8. Horwitz LI, Krumholz HM, Green ML, Huot SJ. Transfers of patients care between house staff on internal medicine wards: a national survey. Arch Intern Med 2006; 166: 1173-7.

9. Arora V, Johnson J, Lovinger D, Humphrey HJ, Meltzer DO. Communication failures in patient sign-out and suggestions for improvement: a critical incident analysis. Qual Saf Health Care 2005; 14: 401-7.

10. Horwitz LI, Moin T, Krumholz HM, Wang L, Bradley EH. Consequences of inadequate sign-out for patient care. Arch Intern Med 2008; 168: 1755-60.

11. Borowitz SM, Waggoner-Fountain LA, Bass EJ, Siedd RM. Adequacy of information transferred at resident sign-out (inhospital handover of care): a prospective survey. Qual Saf Health Care 2008; 17: 6-10.

12. Chassin MR, Becher EC. The wrong patient. Ann Intern Med 2002; 136: 826-33.

13. Van Eaton EG, Horvath KD, Lober WB, Pellegrini CA. Organizing the transfer of patient care information: the development of a computerized resident sign-out system. Surgery 2004; 136: 5-13.

14. Vidyarthi AR, Arora V, Schnipper JL, Wall SD, Wachter RM. Managing discontinuity in academic medical centers: strategies for a safe and effective resident sign-out. J Hosp Med 2006; 1: 257-66.

15. Volpp KG, Grande D. Residents' suggestions for reducing errors in teaching hospitals. N Engl J Med 2003; 348: 851-5.

16. Senge P. La quinta disciplina. Buenos Aires: Granica; 2003.

17. Mukherjee S. Precarious exchange. N Engl J Med 2004; 351: 1822-4.

18. Mitchell R, Regan-Smith M, Fisher MA, Knox I, Lambert DR. A new measure of the cognitive, metacognitive, and experiential aspects of residents' learning. Acad Med 2009; 84: 918-26.

19 Bordage G. Prototypes and semantic qualifiers: from past to present. Med Educ 2007; 41: 1117-21.

20. Bowen JL. Educational strategies to promote clinical diagnostic reasoning. N Engl J Med 2006; 355: 2217-25.

21. Solet DJ, Norvell JM, Rutan GH, Frankel RM. Lost in translation: challenges and opportunities in physician-to-physician communication during patient handoffs. Acad Med 2005; 80: 1094-9.

22. Redelmeter, D. Improving patient care. The cognitive psychology of missed diagnoses. Ann Intern Med 2005; 142: 115-20.

23. Horwitz LI, Moin T, Green ML. Development and implementation of an oral sign-out skills curriculum. J Gen Intern Med 2007; 22: 1470-4. 